\title{
The genetic architecture of divergence between threespine stickleback species
}

Catherine L. Peichel*, Kirsten S. Nereng*, Kenneth A. Ohgi*, Bonnie L. E. Cole*, Pamela F. Colosimo*, C. Alex Buerkle†, Dolph Schluterł \& David M. Kingsley*

*Department of Developmental Biology and Howard Hughes Medical Institute, Stanford University, Stanford, California 94305-5329, USA

†Department of Biology, University of Wisconsin-Eau Claire, Eau Claire, WI 547024004, USA

$\$$ Zoology Department and Centre for Biodiversity, University of British Columbia, Vancouver, British Columbia V6T 1ZT, Canada

The genetic and molecular basis of morphological evolution is poorly understood, particularly in vertebrates. Genetic studies of the differences between naturally occurring vertebrate species have been limited by the expense and difficulty of raising large numbers of animals and the absence of molecular linkage maps for all but a handful of laboratory and domesticated animals. We have developed a genome-wide linkage map for the threespine stickleback (Gasterosteus aculeatus), an extensively studied teleost fish that has undergone rapid divergence and speciation since the melting of glaciers 15,000 years ago ${ }^{1}$. We have used this map to analyze the genetic basis of recently evolved changes in skeletal armor and feeding morphologies seen in the benthic and limnetic stickleback species from Priest Lake, British Columbia. Substantial alterations in spine length, armor plate number, and gill raker number are controlled by genetic factors that map to independent chromosome regions. Further study of these regions will help to 


\section{define the number and type of genetic changes that underlie morphological diversification during vertebrate evolution.}

Threespine sticklebacks provide one of the best-known examples of rapid adaptive radiation in vertebrates. A large number of distinct morphological forms of sticklebacks have evolved following the colonization of newly created coastal streams and lakes at the end of the last Ice Age ${ }^{1}$. In at least 6 lakes in coastal British Columbia, pairs of sympatric stickleback species have been identified. Members of a species pair are adapted to different niches within a lake, with corresponding changes in feeding morphology and defensive armor occurring in parallel in the different lakes (Figure 1) ${ }^{2}$. The benthic species feeds on invertebrates near shore and has a great reduction in the amount of body armor, increased body depth, and a decreased number of gill rakers for filtering ingested food. The limnetic species more closely resembles an ancestral marine fish, with more extensive body armor, a longer and more streamlined body, and an increased number of gill rakers. Despite reproductive isolation between the two species in the wild ${ }^{3-6}$, it is possible to establish productive matings between the two species under laboratory conditions ${ }^{2}$. The resulting F1 hybrids are viable and fertile, making it possible to carry out a formal genetic analysis of the number and location of loci responsible for the adaptive morphological differences between these naturally occurring vertebrate species.

To develop resources for genome-wide linkage mapping in Gasterosteus aculeatus, we used large-scale library screening and sequencing to identify a collection of genomic and cDNA clones containing microsatellite repeat sequences. Initially, we sequenced of $192 \mathrm{~kb}$ of random genomic clones and showed that CA dinucleotides were the most common form of microsatellite in sticklebacks, occurring approximately once every $14 \mathrm{~kb}$. We subsequently screened genomic and cDNA libraries with a $(\mathrm{GT})_{15}$ probe, sequenced 3560 clones, and identified 1176 new microsatellite loci. Primers 
flanking 410 new and 18 previously identified microsatellites ${ }^{7-9}$ were designed and used to type a genetic cross between the benthic and limnetic species from Priest Lake, British Columbia (Figure 1). For this cross, an individual Priest benthic female was mated with a single Priest limnetic male, and a single F1 male $\left(B_{1} L_{1}\right)$ was crossed to a second Priest benthic female $\left(\mathrm{B}_{2} \mathrm{~B}_{3}\right)$ to generate 103 progeny. Of the 281 markers that amplified robust bands from the F1 and benthic parent, 227 (81\%) were polymorphic, and therefore informative, in one or both parents. Higher rates of polymorphism were seen in the F1 male than the benthic female parent (71\% vs. 57\% of 281 markers), consistent with a greater level of genetic diversity between the distinct populations of benthic and limnetic fish than within the benthic population.

The segregation patterns of the 227 informative markers were scored on 92 progeny from the cross, and the 20,884 resulting genotypes were analyzed for linkage using JoinMap software ${ }^{10}$. The markers were ordered into 26 linkage groups covering a total genetic distance of 886 centimorgans, using a conservative LOD threshold of 4.0 (Figure 2). Gasterosteus aculeatus has a total of 21 chromosomes $^{11}$; therefore, we expect that some current linkage groups will collapse with other groups as additional markers are added to the map. Over $96 \%$ of the markers were linked to other markers on the map with an average density of 1 marker per $4 \mathrm{cM}$, suggesting a high probability that the existing markers can be used for genome-wide linkage mapping of interesting traits in many different stickleback populations.

Previous studies have shown benthic and limnetic species have distinct trophic morphologies adapted to feeding on either small invertebrates in the near shore environment, or zooplankton in the open water ${ }^{12,13}$. Limnetic fish have larger eyes, longer snouts and jaws, and more numerous gill rakers, which are morphological adaptations that enhance feeding performance on small zooplankton ${ }^{12}$. To examine the influence of different genetic regions on trophic morphology, we counted the number 
of both long and short gill rakers on the first gill arch of all progeny from the Priest Lake cross (Figure 3b). No major QTL were found influencing the number of long gill rakers in the cross, consistent with previous biometrical studies suggesting that gill raker number may be based on a large number of genes of small effect ${ }^{14,15}$. In contrast, the number of short gill rakers is influenced by two QTL that map to separate linkage groups. Together, these two QTL accounted for nearly two-thirds of the variance in small raker number (Table 1, Figure 2,3c). Evolutionary change in the number of short gill rakers may thus be influenced by genetic effects at a relatively small number of chromosome regions.

The amount of skeletal armor is one of the most striking morphological differences between different stickleback populations, including the benthic and limnetic species pairs. Benthics have reduced armor, often with a reduced or absent first dorsal spine, reduced or absent pelvic spines, and a reduced number of lateral plates (Figure 1). These changes in skeletal armor may be related to the different predation regimes experienced in the near shore and open water environments. Open water populations experience more bird and fish predation, where longer dorsal and pelvic spines appear to offer greater protection ${ }^{15-17}$. In contrast, near shore populations experience predation by insects, an environment in which spine reduction may be advantageous, and may be accompanied by loss of the lateral plates that support the dorsal and pelvic spines ${ }^{17,18}$.

Linkage analysis of spine lengths and lateral plate number in the Priest cross identified QTL influencing the length of the first and second dorsal spine, the pelvic spine, and the number of lateral plates (Table 1, Figure 2,3a,e). These QTL accounted for 17 to $26 \%$ of the total variance in each trait, and mapped to several distinct linkage groups. The locations of the QTL influencing the first and second dorsal spines were completely distinct, suggesting that very similar morphological features can be 
influenced by different genetic regions. In contrast, the length of the second dorsal spine and the pelvic spine were both influenced by QTL that map to a similar region of linkage group VIII. These two spines define the maximal dorsal and ventral extent of stickleback armor (Figure 3d) and are thought to play an important role in defense against gape-limited predators ${ }^{15-17}$. Variation in the length of these functionally related spines may be due to pleiotropic effects of the same locus or to linked genetic factors on linkage group VIII. Co-localization of ecologically important QTL has recently been found in other systems, and may influence patterns of naturally occurring variation, adaptation, and speciation $^{19,20}$.

The variation in length of both the second dorsal spine and the pelvic spine was due primarily to allelic differences segregating within the benthic population (Table 1, Supplementary information). Previous studies have found significant polymorphism for morphological traits within stickleback populations ${ }^{21}$, perhaps maintained by spatial or temporal variation in particular habitats, or contrasting advantages of different skeletal phenotypes at different stages of the stickleback life cycle ${ }^{22,23}$. In contrast, the length of the first dorsal spine and number of lateral plates and short gill rakers were influenced by allelic differences both between and within populations (Table 1, Supplementary information). Although the presence of a limnetic allele was usually associated with an increase in length or number of skeletal elements, this effect was sometimes seen only in the presence of a particular benthic allele. For example, a limnetic allele at the Plate$a$ QTL was associated with higher mean lateral plate number in combination with the $\mathrm{B}_{3}$ benthic allele, but not the $\mathrm{B}_{2}$ benthic allele, suggesting that allelic interactions influence this phenotype (Table 1, Supplementary information). For those traits affected by two distinct QTLs, the presence of limnetic alleles at both QTLs often caused a substantial phenotypic effect. For example, fish with 0,1 , or 2 limnetic alleles at the Plate- $a$ and Plate- $b$ QTL showed an increase from 7.9 to 9.0 to 10.5 in the mean number of lateral plates, and fish with 0,1 , or 2 limnetic alleles at the Spine1- $a$ and Spine1-b QTL 
showed an increase from $0.12 \mathrm{~mm}$ to $1.00 \mathrm{~mm}$ to $1.42 \mathrm{~mm}$ in the mean length of the first dorsal spine (Supplementary information). Therefore, a thirty-three percent difference in armor plate number and a greater than ten fold increase in the size of the first dorsal spine can be influenced by genetic effects at a relatively small number of chromosome regions.

Our results in this vertebrate system are consistent with a number of recent genetic studies in plants and insects suggesting that evolutionary changes between organisms are controlled by genes with a variety of magnitudes of effect, some of which account for a substantial fraction of the variance in particular traits ${ }^{24}$. The small size of the current cross limits our ability to detect additional genes of smaller effect, and could overestimate the contribution of minor QTL ${ }^{25}$. Larger crosses are now needed to identify the full spectrum of genetic changes that contribute to the morphological differences between benthic and limnetic fish, and to narrow the location of the gene or genes within each chromosome interval that contribute to morphological divergence.

The many different chromosome regions that affect specific aspects of skeletal anatomy in sticklebacks reveal a flexible genetic system for independent modification of the size and number of different feeding and armor structures. However, some functionally related traits map to similar chromosome regions, suggesting that genetic linkage or pleiotropy may help account for the covariation in dorsal and pelvic spine lengths previously reported in many stickleback populations ${ }^{15}$. An extensive literature already exists on the dramatic variation in size, morphology, color, and behavior of freshwater sticklebacks around the world ${ }^{1}$. The ease of collecting and crossing such fish in the laboratory, the relatively compact genome size of sticklebacks ${ }^{26}$, the development of genome-wide linkage maps, and the ability to detect QTL for important evolutionary differences provide a particularly favorable system for further molecular studies of the genetic basis of morphological and behavioral changes during vertebrate evolution. 


\section{Methods}

\section{Library construction, screening and sequencing.}

Genomic DNA from a single Paxton lake adult fish was digested with RsaI and fragments from $0.8-1.6 \mathrm{~kb}$ were cloned into the EcoRV site of pBluescriptSK $(+)$. An oligo(dT) primed cDNA library was constructed in lambda ZAP Express by Stratagene using RNA isolated from the head and internal organs of two Salinas River, CA adults. Colony and phage lifts were hybridized with an end-labeled oligonucleotide $(\mathrm{GT})_{15}$ in 4X SSPE, $1 \mathrm{X}$ Denhardt's, and $1 \%$ SDS at $60^{\circ} \mathrm{C}$ overnight, and then washed $2 \mathrm{X} 10$ minutes in $0.1 \mathrm{X} \mathrm{SSC} ; 0.1 \% \mathrm{SDS}$ at $60^{\circ} \mathrm{C}$. All positive clones were grown overnight in 96 well plates, and plasmid DNA was prepared using the QIAprep96 Turbo miniprep (QIAGEN). Each clone was sequenced with a T3 and a T7 primer on a 96 lane gel on an $\mathrm{ABI} 377$ sequencer. Clones containing a microsatellite repeat were analyzed with Primer $3^{27}$ to identify primer pairs flanking the microsatellite. Primer pairs were picked to have melting temperatures between $55-65^{\circ} \mathrm{C}$ and to give products between $100-250$ bp. Forward primer plates were labeled with one of three 5' phosphoramidite flourescent conjugates: 6-fam (blue), 6-hex (yellow) or tet (green).

\section{Genotyping.}

All PCR reactions were carried out in an PTC-200 DNA Engine thermocycler (MJ Research) in $10 \mu 1$ reactions containing $0.5 \mu \mathrm{M}$ of each primer, $5 \mathrm{ng}$ DNA, $0.25 \mathrm{mM}$ dNTPs (Pharmacia), 1.5 $\mathrm{mM} \mathrm{MgCl}_{2}$, and 0.25 Units Taq polymerase (PE Applied Biosystems). The cycling conditions for all primer pairs were 1 cycle of $95^{\circ} \mathrm{C}$ for 1 ' $45^{\prime \prime}$, $56^{\circ} \mathrm{C}$ for $45^{\prime \prime}$, and $72^{\circ} \mathrm{C}$ for $45^{\prime \prime} ; 5$ cycles of $94^{\circ} \mathrm{C}$ for $45^{\prime \prime}, 56^{\circ} \mathrm{C}$ for $45^{\prime \prime}$, and $72^{\circ} \mathrm{C}$ for 
$45^{\prime}$; and 30 cycles of $90^{\circ} \mathrm{C}$ for $45^{\prime \prime}, 56^{\circ} \mathrm{C}$ for $45^{\prime}$, and $72^{\circ} \mathrm{C}$ for $45^{\prime}$, followed by a final cycle of $72^{\circ} \mathrm{C}$ for 5 minutes. PCR products from 3 to 6 different primer pairs were then pooled and analyzed on a 96 lane gel on an ABI377 with Gene Scan 2.1 software and GENESCAN-500 TAMRA (PE Applied Biosystems) used as internal size standard.

\section{Linkage map construction.}

A genetic linkage map was created using JoinMap version $2.0^{10}$ on a locus file containing genotypes of 227 microsatellite loci in 92 backcross progeny, with the population type set for segregation of up to 4 alleles per locus (cross-pollinator). The JMGRP module was used with a LOD threshold of 4.0 to assign 219 of the 227 loci to 26 linkage groups. The JMREC module was then used on each of the linkage groups to determine phase information for each locus. For each linkage group, a map was created with the JMMAP module: Kosambi mapping function, LOD threshold of 0.001, REC threshold of 0.499 , jump threshold of 5.0, triplet value of 5.0, and no fixed order. A ripple was performed after all markers on the linkage group were added to the map.

\section{Morphological analysis.}

Fish were fixed in $10 \%$ buffered formalin for at least 1 week, placed in $\mathrm{dH}_{2} \mathrm{O}$ for 24 hours, stained with $0.008 \%$ alizarin red in $1 \% \mathrm{KOH}$ for 24 hours, placed in $\mathrm{dH}_{2} \mathrm{O}$ for 24 hours, and placed in 37\% isopropyl alcohol for final storage. Measurements were done with Vernier calipers accurate to $0.02 \mathrm{~mm}$. Lateral plates were counted on both sides of the body, and the number of long and short gill rakers were counted on the left side of the first gill arch.

\section{QTL mapping.}

All morphological traits were analyzed with MapQTL $3.0^{28}$ using the interval mapping method which fits a single QTL model based on four possible segregating genotypes 
and does not assume a particular model of relationship between benthic and limnetic alleles. The parameter used were: mapping step size of 5.0, maximum of 200 iterations, and functional tolerance value of $1.0 \mathrm{e}^{-8}$. A maximum of 5 flanking markers were used to resolve incomplete genotypes. Significance thresholds for linkage were chosen using conservative criteria for genome wide linkage mapping in non-inbred individuals: suggestive linkage of $L O D \geq 3.2$, significant linkage of $L O D \geq 4.5^{29}$. Significance thresholds were confirmed by permutation tests in MapQTL 4.0, with a genome-wide significance level of $\alpha=0.05, \mathrm{n}=1000$ for significant linkages and a chromosome-wide significance level of $\alpha=0.05, \mathrm{n}=1000$ for suggestive linkages. Suggestive loci were only reported for those traits which were also influenced by one or more significant QTL. Multiple QTL model (MQM) mapping with initial QTL did not change the results. Calculation of the percent of phenotypic variance explained by a QTL was done in MapQTL 3.0 based on the population variance found within the progeny of the cross.

\section{References}

1. Bell, M. A. \& Foster, S. A. The Evolutionary Biology of the Threespine Stickleback (Oxford Science, New York, 1994).

2. McPhail, J. D. Speciation and the evolution of reproductive isolation. in The Evolutionary Biology of the Threespine Stickleback (eds Bell, M.A. \& Foster, S. A.) 399-437 (Oxford University Press, New York, 1994).

3. Ridgway, M. S. \& McPhail, J. D. Ecology and evolution of sympatric sticklebacks (Gasterosteus): mate choice and reproductive isolation in the Enos Lake species pair. Can. J. Zool. 62, 1813-1818 (1984). 
4. Nagel, L. \& Schluter, D. Body size, natural selection, and speciation in sticklebacks. Evolution 52, 209-218 (1998).

5. Hatfield, T. \& Schluter, D. Ecological speciation in sticklebacks: environmentdependent hybrid fitness. Evolution 53, 866-873 (1999).

6. Vamosi, S. M. \& Schluter, D. Sexual selection against hybrids between sympatric stickleback species: evidence from a field experiment. Evolution 53, 874-879 (1999).

7. Rico, C., Zadworny, D., Kuhnlein, U. \& Fitzgerald, G. J. Characterization of hypervariable microsatellite loci in the threespine stickleback Gasterosteus aculeatus. Mol. Ecol. 2, 271-272 (1993).

8. Taylor, E. B. Microsatellites isolated from the threespine stickleback Gasterosteus aculeatus. Mol. Ecol. 7, 925-931 (1998).

9. Largiader, C. R., Fries, V., Kobler, B. \& Bakker, C. M. Isolation and characterization of microsatellite loci from the three-spined stickleback (Gasterosteus aculeatus L.). Mol. Ecol. 8, 342-344 (1999).

10. Stam, P. \& Van Ooijen. JoinMap (tm) version 2.0: Software for the calculation of genetic linkage maps. CPRO-DLO, Wageningen (1995).

11. Chen, T.-R. \& Reisman, H. M. A comparative study of the North American species of sticklebacks (Teleostei: Gasterosteidae). Cytogenetics 9, 321-332 (1970).

12. Bentzen, P. \& McPhail, J. D. Ecology and evolution of sympatric sticklebacks (Gasterosteus): specialization for alternative trophic niches in the Enos Lake species pair. Can. J. Zool. 62, 2280-2286 (1984). 
13. Schluter, D. Adaptive radiation in sticklebacks: size, shape, and habitat use efficiency. Ecology 74, 699-709 (1993).

14. Hatfield, T. Genetic divergence in adaptive characters between sympatric species of stickleback. Am. Nat. 149, 1009-1029 (1997).

15. Hagen, D. W. \& Gilbertson, L. G. Geographic variation and environmental selection in Gasterosteus aculeatus L. in the Pacific Northwest, America. Evolution 26, 32-51 (1972).

16. Moodie, G. E. E. Predation, natural selection and adaptation in an unusual threespine stickleback. Heredity 28, 155-167 (1972).

17. Reimchen, T. E. Spine deficiency and polymorphism in a population of (Gasterosteus aculeatus): an adaptation to predators? Can. J. Zool. 58, 1232-1244 (1980).

18. Reimchen, T. E. Structural relationship between spines and lateral plates in threespine stickleback (Gasterosteus aculeatus). Evolution 37, 931-946 (1983).

19. Hawthorne, D. J. \& Via, S. Genetic linkage of ecological specialization and reproductive isolation in pea aphids. Nature 412, 904-907 (2001).

20. Lynch, M. \& Walsh, J. B. Genetics and Analysis of Quantitative Traits (Sinauer, Massachusetts, 1998).

21. Ahn, D. \& Gibson, G. Axial variation in the threespine stickleback: genetic and environmental factors. Evolution and Development 1, 100-112 (1999).

22. Reimchen, T. E. Predator-induced cyclical changes in lateral plate frequencies of Gasterosteus. Behavior 132, 1079-1094 (1995) 
23. Swain, D.P. Selective predation for vertebral phenotype in Gasterosteus aculeatus: reversal in the direction of selection at different larval sizes. Evolution 46, 998-1013 (1992).

24. Orr, H. A. The genetics of species differences. Trends Ecol. Evol. 16, 343-350 (2001).

25. Beavis, W. D. QTL Analyses: Power, Precision, and Accuracy. in Molecular Dissection of Complex Traits (ed Paterson, A. H.) 145-162 (CRC Press, Boca Raton, 1998).

26. Hinegardner, R. Evolution of cellular DNA content in teleost fishes. Am. Nat. 102, $517-523(1968)$.

27. Rozen, S. \& Skaletsky, H. J. Primer3. Code available at http://wwwgenome.wi.mit.edu/genome software/other/primer3.html (1997).

28. VanOoijen, J. W. \& Maliepard, C. MapQTL (tm) version 3.0: Software for the calculation of QTL positions on genetic maps. CPRO-DLO, Wageningen. (1996).

29. VanOoijen, J. W. LOD significance thresholds for QTL analysis in experimental populations of diploid species. Heredity 83, 613-624 (1999).

30. Bell, M. A. Evolutionary Phenetics and Genetics: The Threespine Stickleback, Gasterosteus aculeatus, and Related Species. in Evolutionary Genetics of Fishes (ed Turner, B. J.) 431-528 (Plenum Press, New York, 1984).

Supplementary information is available on Nature's World-Wide Web site (http://www.nature.com) or as paper copy from the London editorial office of Nature.

Acknowledgements

We would like to thank Shawn Anderson, Rachel Melzer, Karen Tsui, Caroline Uhlick and Rebecca Vega for technical assistance. D. S. was supported by a Natural Sciences and Engineering Research Council of 
Canada research grant. C.L.P. is a research associate and D.M.K is an assistant investigator of the Howard Hughes Medical Institute.

Correspondence and requests for materials should be addressed to D.M.K. (email:kingsley@cmgm.stanford.edu). Genbank accession numbers for stickleback microsatellite markers are G72126 through G72334. 
Figure 1. Representative benthic and limnetic fish from Priest Lake, British Columbia are stained with alizarin red to highlight bone. The benthic fish are larger, more deep bodied, and have fewer bony lateral plates than the limnetics. Scale bars, $5 \mathrm{~mm}$.

Figure 2. Genetic linkage map of Gasterosteus aculeatus. Each linkage group has been assigned a Roman numeral in order of total genetic length. Microsatellite loci identified at Stanford are designated with a prefix of Stn, followed by a number which is based on its serial position in the initial map, Twelve previously published microsatellites are also included on the map ${ }^{7-9}$. The map locations of QTL affecting feeding morphology and skeletal armor are shown in red.

Figure 3. Mapping of morphological traits in the Priest Lake cross. Morphological measurements and traits for which significant QTL were found: a, d, skeletal armor shown in profile (a) or cross-section (d, adapted from reference 30 ); b, gill raker numbers. The LOD scores (Y-axis) were graphed relative to position in $\mathrm{cM}$ along the linkage group (X-axis): $\mathbf{c}$, number of small gill rakers; e, armor traits. Dots indicate the LOD score for markers on the linkage group. The lines were drawn by plotting the LOD scores calculated by MapQTL at each marker as well as at $5.0 \mathrm{cM}$ intervals along the linkage group. A graph is not shown for the Plate-b locus on LG XXVI, which consists of two nonrecombinant markers. 
Kingsley_Table1

Table 1. Location and magnitude of effect for QTLs

Trait

Locus name LG

LOD

PVE

Phenotype means

\section{Feeding modifications}

Gill raker number

$\begin{array}{llll}\text { Raker\#-a } & \text { XI } & 5.5^{*} & 26 \% \\ \text { Raker\#-b } & \text { XVI } & 6.8^{*} & 37 \%\end{array}$

$\mathrm{L}_{1} \mathrm{~B}_{2} \quad \mathrm{~B}_{1} \mathrm{~B}_{2} \quad \mathrm{~L}_{1} \mathrm{~B}_{3} \quad \mathrm{~B}_{1} \mathrm{~B}_{3}$

\section{Body armor}

Lateral plate number

Plate-a XIII

$5.5^{*}$

$26 \%$

$4.6^{*}$

$22 \%$

Dorsal spine 1 length

Plate-b

$\mathrm{XXVI}$

Spine1-a ।

$4.7^{*}$

$21 \%$

Spine1-b II

3.6

$17 \%$

Dorsal spine 2 length

Spine2-a VIII

$4.5^{*}$

$22 \%$

3.4

$17 \%$

Pelvic spine length

Spine2-b XI

$4.5^{*}$

$25 \%$

$\begin{array}{rrrl}15.4 & 14.3 & 13.8 & 13.8 \text { rakers }^{\dagger \S \#} \\ 14.9 & 13.2 & 14.4 & 14.3 \text { rakers }^{\dagger \#} \\ 8.5 & 8.6 & 10.6 & 8.4 \text { plates }^{\dagger \S \#} \\ 10.0 & 8.2 & 9.6 & 8.0 \text { plates }^{\dagger} \\ 1.53 & 0.49 & 1.00 & 0.57 \mathrm{~mm}^{\dagger} \\ 1.42 & 0.73 & 0.97 & 0.13 \mathrm{~mm}^{\dagger \S} \\ 2.72 & 2.59 & 2.31 & 2.35 \mathrm{~mm}^{\S} \\ 2.57 & 2.65 & 2.22 & 2.39 \mathrm{~mm}^{\S} \\ 3.35 & 3.41 & 2.91 & 2.84 \mathrm{~mm}^{\S}\end{array}$

For each QTL detected, the linkage group (LG), maximum LOD score, and percent of the phenotypic variance explained (PVE) are indicated. QTLs were scored as significant (asterisks) or suggestive based on conservative recommendations for genome wide linkage mapping ${ }^{29}$, and by permutation testing for each trait. The genome-wide LOD significance thresholds are 4.2 for gill raker number and pelvic spine length, and 4.3 for plate number and dorsal spine length. The chromosome-wide LOD suggestive thresholds are 2.9 for length of dorsal spine 1 on LG II, and 2.6 for length of dorsal spine 2 on LG XI. Mean phenotypic values of each trait were also calculated for those progeny that inherited either all benthic alleles $\left(B_{1} B_{2}\right.$ and $\left.B_{1} B_{3}\right)$, or both limnetic and benthic alleles $\left(L_{1} B_{2}\right.$ and $\left.L_{1} B_{3}\right)$ at the most closely linked microsatellite. Significant differences between phenotype means are the estimated effect of alternate alleles inherited from the $F 1$ male parent $\left(L_{1}\right.$ or $B_{1}$, the between species effect $\left.{ }^{\dagger}\right)$, alleles inherited from the benthic female parent $\left(B_{2}\right.$ or $B_{3}$,

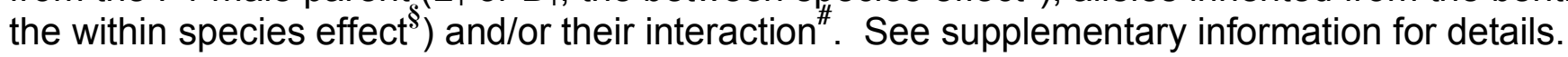




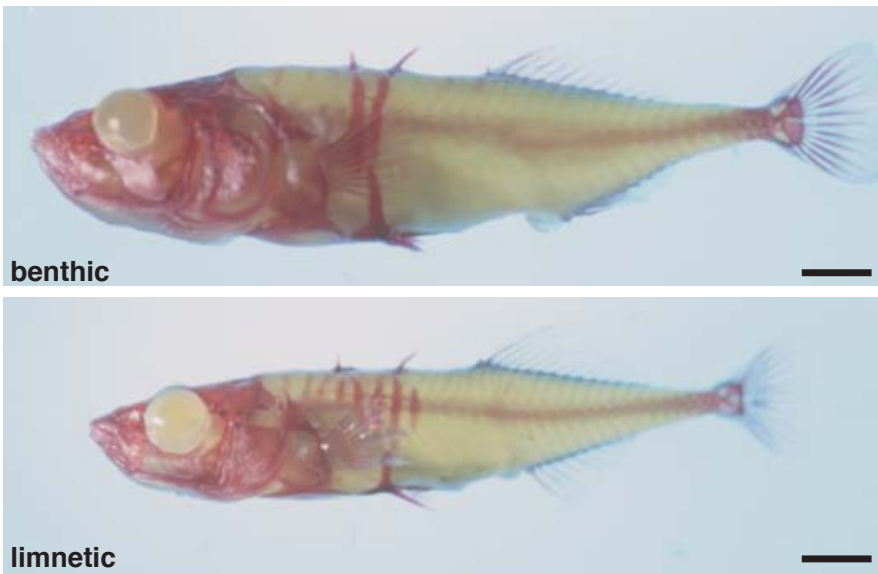




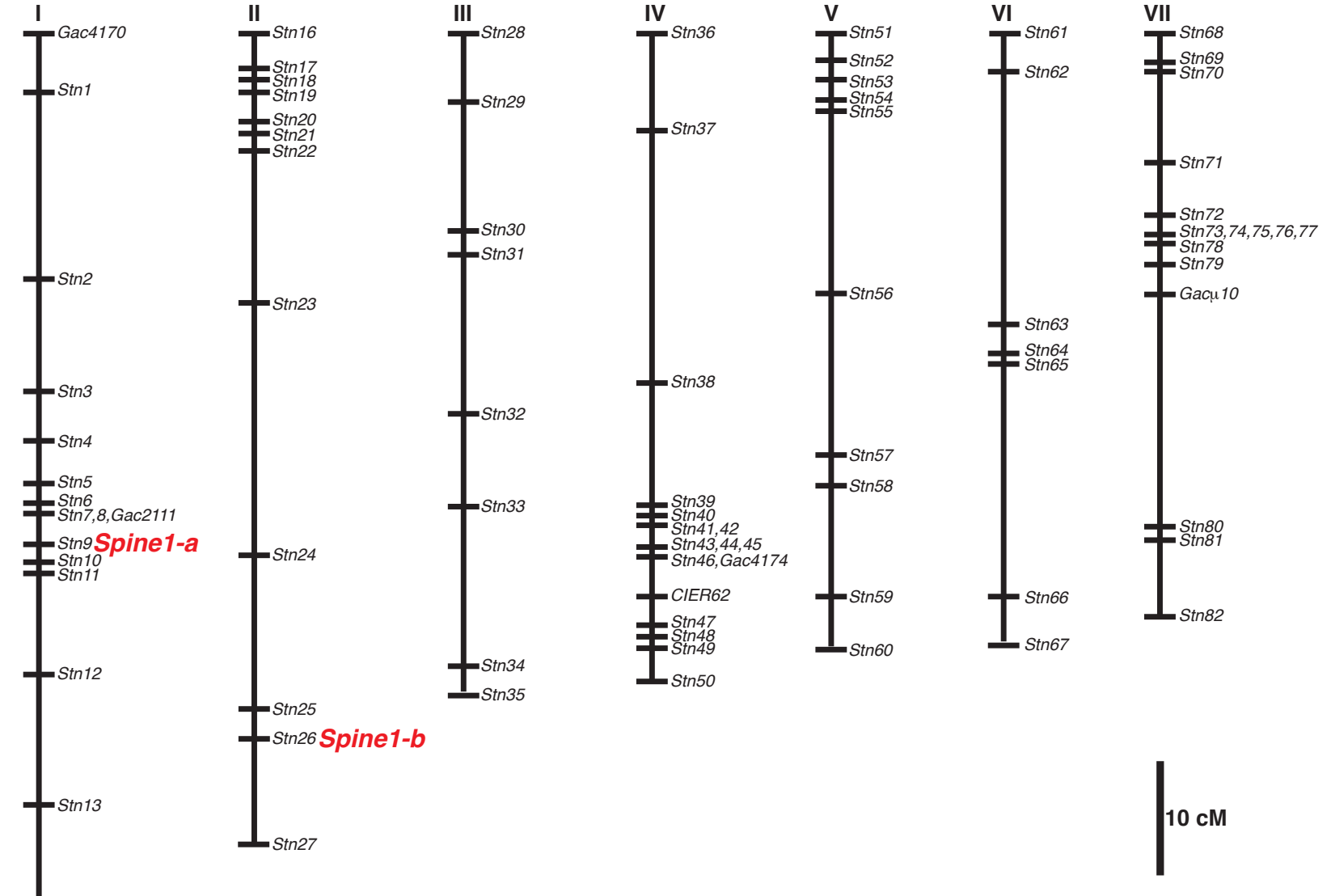

Stn 15
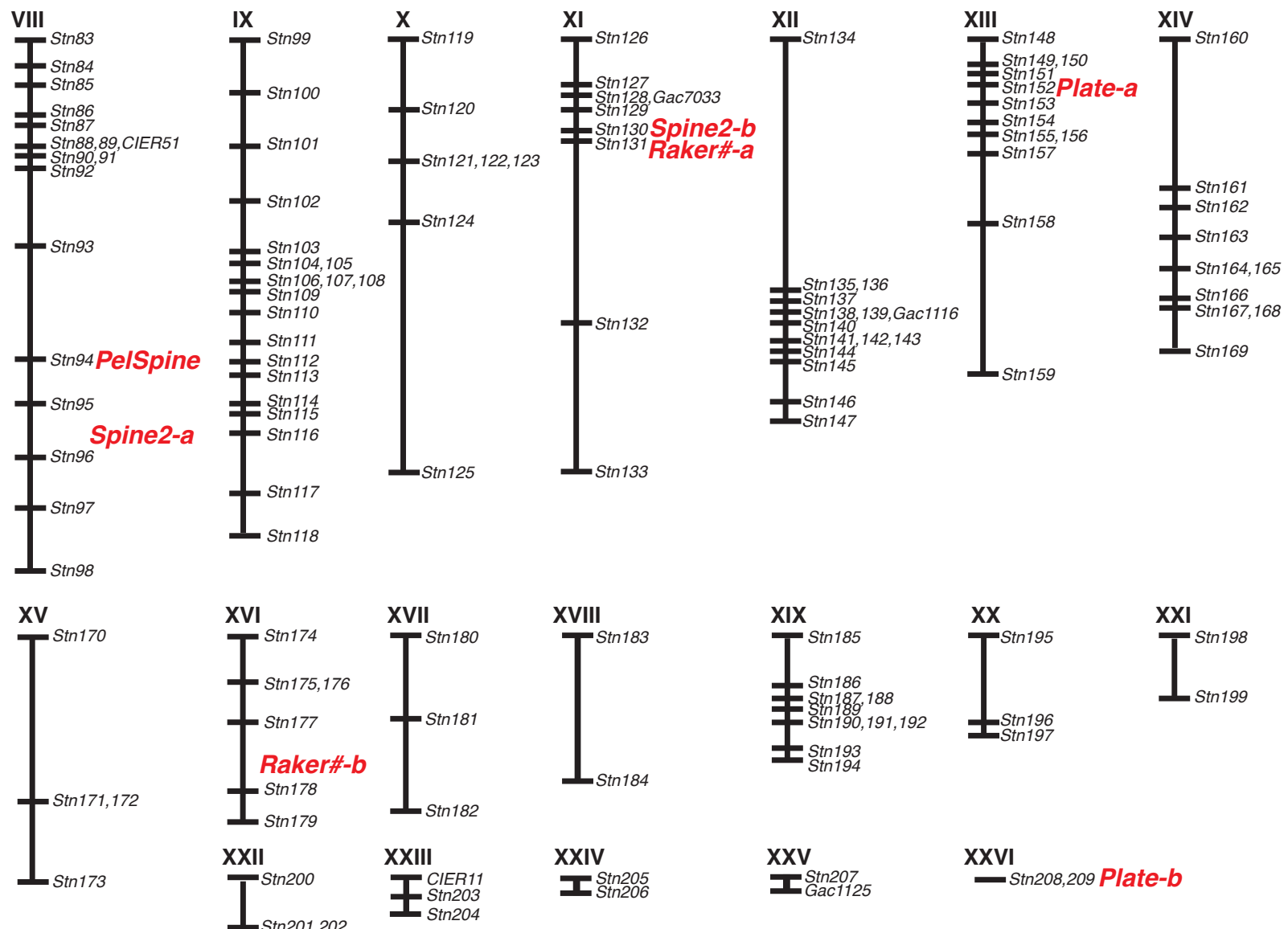

$\mathbf{X X}^{-}$

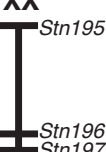

XX

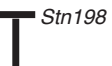

- $\begin{aligned} & \text { Stn196 } \\ & \text { Stn197 }\end{aligned}$

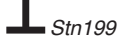

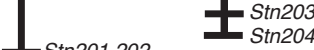

XXV $\mathbf{I}_{\mathrm{Gac} 125}^{\operatorname{Stn} 207}$

XXVI 

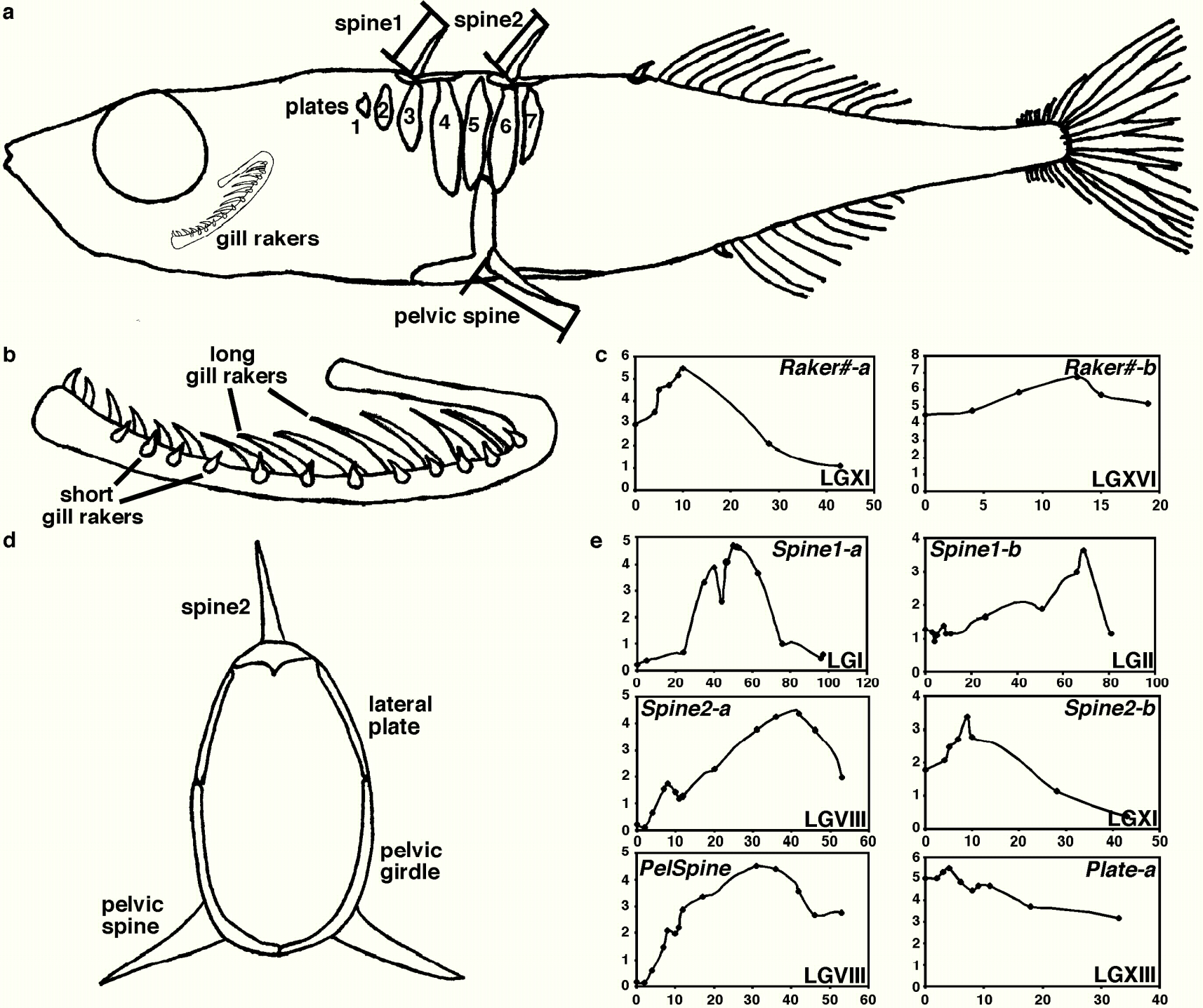
Kingsley_supplementary information

\section{Supplementary Table 2. Phenotype means of fish with different allele combinations at most closely linked microsatellite}

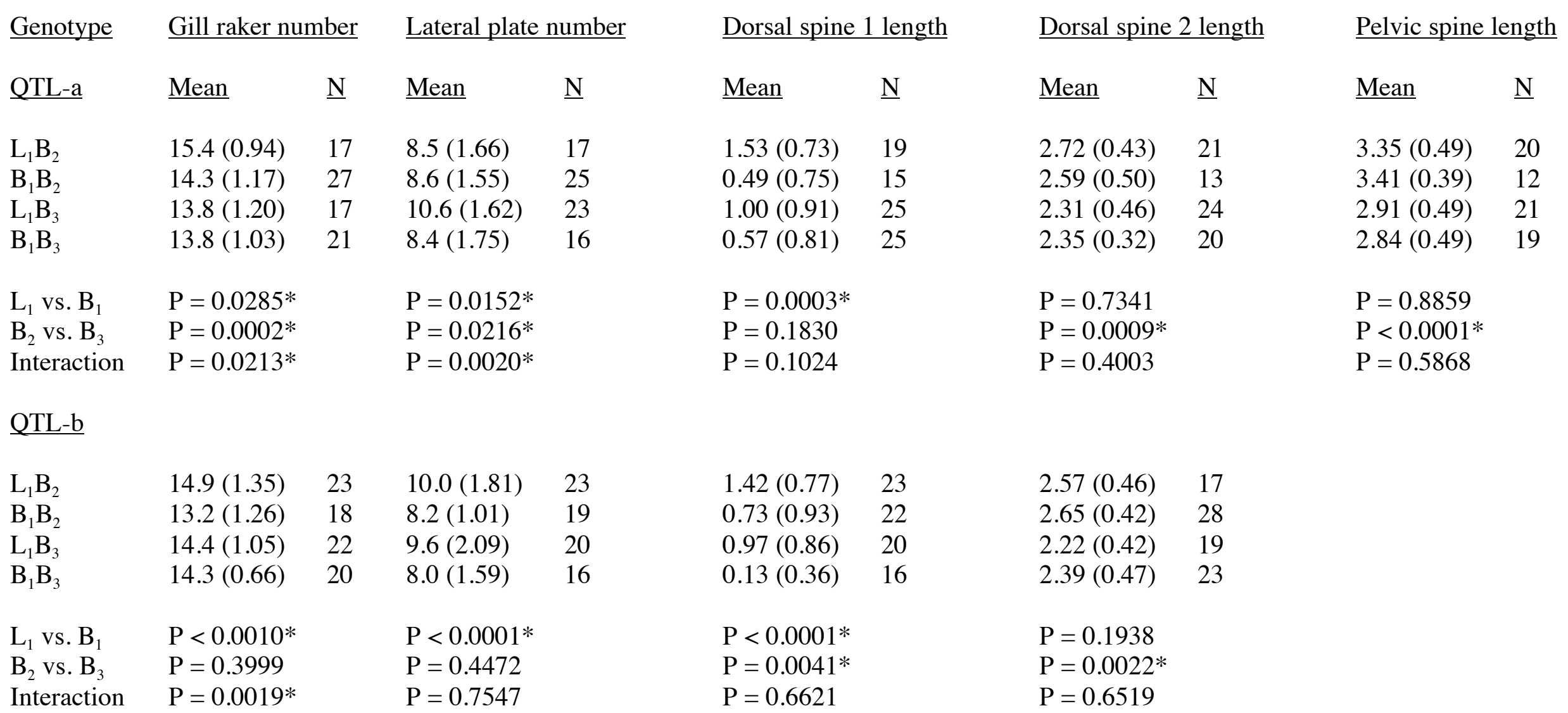

Mean phenotype, standard deviation (in parenthesis), and sample size (N) of animals having a particular allele combination at the microsatellite marker nearest each QTL. Contribution of within and between species genetic variation to differences between phenotype means was estimated using two-factor ANOVA. The first (between species) factor represented the effect on mean phenotype of alternate alleles inherited from the F1 male parent $\left(\mathrm{L}_{1}\right.$ vs. $\left.\mathrm{B}_{1}\right)$. The second (within species) factor represented the effect of alternate alleles inherited from the benthic female parent $\left(\mathrm{B}_{2}\right.$ vs. $\left.\mathrm{B}_{3}\right)$. $\mathrm{P}-$ values for these tests are based on the two-factor model lacking an interaction term. Interaction of within and between species effects was subsequently tested by determining whether inclusion of the interaction term significantly improved the fit to the data. $* \mathrm{P}<0.05$. 
Supplementary Table 3. Estimated effect of increased number of limnetic alleles across pairs of QTLs.

\section{Genotype}

QTL-a QTL-b

$\mathrm{B}_{1} \mathrm{~B}_{2} \quad \mathrm{~B}_{1} \mathrm{~B}_{2}$

$\mathrm{B}_{1} \mathrm{~B}_{2} \quad \mathrm{~B}_{1} \mathrm{~B}_{3}$

$\mathrm{B}_{1} \mathrm{~B}_{3} \quad \mathrm{~B}_{1} \mathrm{~B}_{2}$

$\mathrm{B}_{1} \mathrm{~B}_{3} \quad \mathrm{~B}_{1} \mathrm{~B}_{3}$

0 limnetic alleles

$\mathrm{L}_{1} \mathrm{~B}_{2} \quad \mathrm{~B}_{1} \mathrm{~B}_{2}$

$\mathrm{L}_{1} \mathrm{~B}_{2} \quad \mathrm{~B}_{1} \mathrm{~B}_{3}$

$\mathrm{L}_{1} \mathrm{~B}_{3} \quad \mathrm{~B}_{1} \mathrm{~B}_{2}$

$\mathrm{L}_{1} \mathrm{~B}_{3} \quad \mathrm{~B}_{1} \mathrm{~B}_{3}$

$\mathrm{B}_{1} \mathrm{~B}_{2} \quad \mathrm{~L}_{1} \mathrm{~B}_{2}$

$\mathrm{B}_{1} \mathrm{~B}_{3} \quad \mathrm{~L}_{1} \mathrm{~B}_{2}$

$\mathrm{B}_{1} \mathrm{~B}_{2} \quad \mathrm{~L}_{1} \mathrm{~B}_{3}$

$\mathrm{B}_{1} \mathrm{~B}_{3} \quad \mathrm{~L}_{1} \mathrm{~B}_{3}$

1 limnetic allele

$\mathrm{L}_{1} \mathrm{~B}_{2} \quad \mathrm{~L}_{1} \mathrm{~B}_{2}$

$\mathrm{L}_{1} \mathrm{~B}_{2} \quad \mathrm{~L}_{1} \mathrm{~B}_{3}$

$\mathrm{L}_{1} \mathrm{~B}_{3} \quad \mathrm{~L}_{1} \mathrm{~B}_{2}$

$\mathrm{L}_{1} \mathrm{~B}_{3} \quad \mathrm{~L}_{1} \mathrm{~B}_{3}$

2 limnetic alleles

\section{Gill raker number}

$\begin{array}{ll}\text { Mean } & \underline{N} \\ 13.6(1.39) & 7 \\ 14.4(0.79) & 7 \\ 13.2(0.45) & 5 \\ 14.0(0.63) & 6 \\ \mathbf{1 3 . 8}(\mathbf{0 . 9 9}) & \mathbf{2 5}\end{array}$

0

$14.8(0.50) \quad 4$

$13.2(1.48) \quad 5$

$14.0(0.00) \quad 3$

$\begin{array}{ll}15.0(1.41) & 6 \\ 13.0(1.00) & 3 \\ 14.3(0.82) & 6 \\ 14.7(1.21) & 6 \\ \mathbf{1 4 . 2 ( 1 . 2 3 )} & \mathbf{3 3}\end{array}$

$15.7(1.11) \quad 9$

$15.3(0.58) \quad 3$

$14.8(0.50) \quad 4$

$13.8(1.26) \quad 4$

$15.1(1.19) \quad 20$

$\mathrm{P}<0.0001 *$

\section{$\underline{\text { Lateral plate number }}$}

$\begin{array}{ll}\text { Mean } & \underline{N} \\ 8.2(0.84) & 5 \\ 7.9(1.36) & 8 \\ 7.3(0.96) & 4 \\ 8.0(0.00) & 3 \\ \mathbf{7 . 9 ( 1 . 0 4 )} & \mathbf{2 0}\end{array}$

$8.6(1.13) \quad 7$

$7.7(1.53) \quad 3$

$8.5(0.71) \quad 2$

12.0

$\begin{array}{ll}9.1(1.86) & 7 \\ 9.0(2.65) & 3 \\ 9.6(1.52) & 5 \\ 9.3(2.50) & 4 \\ \mathbf{9 . 0 ( 1 . 7 4 )} & \mathbf{3 2} \\ & \\ 11.0(1.00) & 3 \\ 7.0(0.00) & 2 \\ 10.6(1.51) & 10 \\ 11.2(1.72) & 6 \\ \mathbf{1 0 . 5 ( 1 . 8 1 )} & \mathbf{2 1}\end{array}$

$\mathrm{P}<0.0001 *$

\section{Dorsal spine 1 length}

$\begin{array}{ll}\text { Mean } & \underline{N} \\ 0.31(0.62) & 4 \\ 0.00(0.00) & 4 \\ 0.00(0.00) & 6 \\ 0.18(0.43) & 6 \\ \mathbf{0 . 1 2}(\mathbf{0 . 3 6}) & \mathbf{2 0} \\ & \\ 1.52(0.93) & 5 \\ 0.00 & 1 \\ 1.11(1.07) & 5 \\ 0.26(0.51) & 4 \\ & \\ 1.64(0.65) & 2 \\ 1.37(0.91) & 5 \\ 0.39(0.68) & 3 \\ 0.86(0.91) & 6 \\ \mathbf{1 . 0 0}(\mathbf{0 . 9 1}) & \mathbf{3 1} \\ & \\ 1.80(0.24) & 8 \\ 1.76(0.36) & 3 \\ 1.20(0.91) & 6 \\ 0.95(0.81) & 6 \\ \mathbf{1 . 4 2}(\mathbf{0 . 7 4}) & \mathbf{2 3} \\ & \\ \mathbf{P}<\mathbf{0 . 0 0 0 1} * & \end{array}$

\section{Dorsal spine 2 length}

$\begin{array}{ll}\text { Mean } & \underline{N} \\ 2.66(0.85) & 4 \\ 3.12 & 1 \\ 2.26(0.27) & 5 \\ 2.43(0.28) & 8 \\ \mathbf{2 . 4 7}(\mathbf{0 . 4 7 )} & \mathbf{1 8} \\ & \\ 2.53(0.39) & 7 \\ 2.92(0.54) & 8 \\ 2.32(0.27) & 5 \\ 2.53(0.32) & 7 \\ & \\ 2.40(0.18) & 5 \\ 2.30(0.41) & 5 \\ 2.86 & 1 \\ 2.35(0.58) & 2 \\ \mathbf{2 . 5 3}(\mathbf{0 . 4 2}) & \mathbf{4 0} \\ 2.82(0.11) & 2 \\ 2.61(0.32) & 4 \\ 1.80(0.29) & 4 \\ 2.48(0.61) & 6 \\ \mathbf{2 . 3 9}(\mathbf{0 . 5 4}) & \mathbf{1 6}\end{array}$

$P=0.6083$

Phenotype means, standard deviations (in parentheses) and sample size of animals having 0,1 or 2 limnetic alleles at the microsatellite markers nearest each of the two detected QTLs affecting each trait. P-values indicate significance of the relationship between number of limnetic alleles and phenotype, tested using linear regression. P-values were similar when categories of individuals having 0,1 , or 2 limnetic alleles were compared using single-factor ANOVA instead. Pelvic spine length is not included because only a single QTL was detected. ${ }^{*} \mathrm{P}<0.05$. 